\title{
In Vivo Role of a Potassium Channel-Binding Protein in Regulating Neuronal Excitability and Behavior
}

\author{
Mohammad Shahidullah, ${ }^{\star}$ Smitha Reddy, ${ }^{\star}$ Hong Fei, and Irwin B. Levitan \\ Department of Neuroscience, University of Pennsylvania School of Medicine, Philadelphia, Pennsylvania 19104
}

\begin{abstract}
Molecular details of ion channel interactions with modulatory subunits have been investigated widely in transfected cells, but the physiological roles of ion channel modulatory protein complexes in native neurons remain largely unexplored. The Drosophila largeconductance calcium-activated potassium channel (dSlo) binds to and is modulated by its binding partner Slob. We have constructed flies in which Slob expression is manipulated by P-element mutagenesis, or by transgenic expression of Slob protein or Slob-RNAi. In vivo recordings of both macroscopic and single dSlo channel currents in identified neurosecretory neurons in the pars intercerebralis (PI) region of the Drosophila brain reveal that whole-cell potassium current and properties of single dSlo channels are modulated by Slob expression level. Furthermore, Slob genotype influences action potential duration in vivo. This unprecedented combination of currentclamp, macroscopic-current, and single-channel recordings from neurons in brains of living flies defines a critical role for an ion channel modulatory protein complex in the control of neuronal excitability. We show further that Slob-null flies exhibit significantly longer lifespan than controls under conditions of complete food deprivation. Crosses with deficiency lines demonstrate that this enhanced resistance to starvation-induced death maps close to the slob locus. Together, these results indicate that Slob may serve a novel regulatory function in feeding behavior, possibly by influencing the excitability of the PI neurons.
\end{abstract}

\section{Introduction}

Many ion channels exist as part of a dynamic regulatory protein complex, which may also contain auxiliary subunits and signaling proteins that modulate channel function (Levitan, 2006). Among the channels subject to such modulation is the largeconductance voltage- and calcium-activated potassium channel that is ubiquitous in neurons and other cell types (Vergara et al., 1998). This channel, often called BK (for big potassium) or Maxi $\mathrm{K}$ because of its uniquely large single-channel conductance, participates prominently in action potential repolarization (Shao et al., 1999) and regulates neurotransmitter release from presynaptic terminals (Raffaelli et al., 2004). In the fruit fly Drosophila, mutations in the slo gene lead to a loss of calcium-dependent potassium current (Elkins et al., 1986), and the dSlo protein encoded by this gene is the pore-forming $\alpha$ subunit of a BK channel (Atkinson et al., 1991; Adelman et al., 1992). Extensive alternative splicing (Adelman et al., 1992) gives rise to multiple dSlo channels with diverse functional properties (Lagrutta et al., 1994).

\footnotetext{
Received June 25, 2009; revised Aug. 6, 2009; accepted Sept. 15, 2009.

This work was supported by a grant to I.B.L. from the National Institutes of Health. We are grateful to Gunter Korge for providing the Mai301, Kurs45, and Kurs58 lines, Eric Rulifson for the dilp2-GAL4,UAS-GFP line, and Ennio Giordano for the Sym-pUAST vector. We thank Greg Bashaw and Amita Sehgal for expert advice on fly genetics, and Wei Peter Zhang for help with statistical analysis. We also thank Levitan laboratory members for critical comments on this manuscript, and members of Amita Sehgal's laboratory, especially Sam Johnson, for fly reagents, helpful suggestions, and discussions.

${ }^{*}$ M.S and S.R. contributed equally to this work.

Correspondence should be addressed to Irwin B. Levitan, Department of Neuroscience, University of Pennsylvania School of Medicine, 3450 Hamilton Walk, Philadelphia, PA 19104. E-mail: levitani@mail.med.upenn.edu.

Smitha Reddy's present address: Department of Medical Oncology, Fox Chase Cancer Center, Philadelphia, PA 19111.

DOI:10.1523/JNEUROSCI.3024-09.2009

Copyright $\odot 2009$ Society for Neuroscience $\quad 0270-6474 / 09 / 2913328-10 \$ 15.00 / 0$
}

dSlo is also modulated by the binding of an auxiliary protein called Slob (for Slo-binding) (Schopperle et al., 1998; Zeng et al., 2006). Alternative splicing gives rise to multiple Slob proteins, which modulate dSlo in different ways (Jaramillo et al., 2006), thereby contributing further to functional diversity. Like dSlo, Slob protein is expressed widely in the Drosophila brain and eye, and it is especially prominent in neurosecretory neurons of the dorsomedial pars intercerebralis (PI) region of the cortex (Jaramillo et al., 2004). These neurons synthesize and secrete Drosophila insulin-like peptides (DILPs), important regulators of hemolymph sugar levels and metabolism (Rulifson et al., 2002; Kim and Rulifson, 2004). Slob expression in whole fly heads as well as in the PI neurons themselves is under the control of the circadian clock (Jaramillo et al., 2004). This finding implies that the modulatory action of Slob in PI and other neurons may itself be subject to dynamic regulation.

The interactions of modulatory auxiliary subunits with ion channels typically are studied by expression in heterologous host cells. While much has been learned from such studies (Levitan, 2006), they lack the comfort of physiological reality, and there is little information available about the roles ion channel modulatory protein complexes might play in native neurons. To gain insight into the in vivo roles of Slob, we used two complementary approaches, P-element mutagenesis and transgenic RNAi, to generate flies with absent or reduced Slob expression. We also used targeted expression of Slob57, the predominant Slob isoform, to increase the level of Slob in the PI neurons of wild-type flies and to rescue the Slob-null and Slob-RNAi knock-out/ knockdown flies. In vivo recordings of action potentials, wholecell potassium currents and single dSlo channels in PI neurons from these various fly lines demonstrate that Slob, via its interac- 


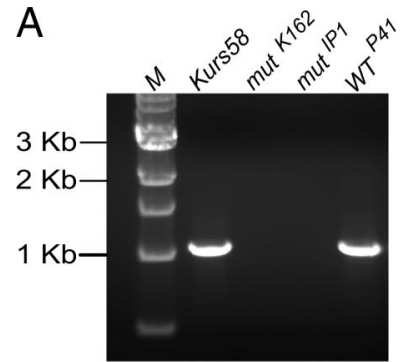

B
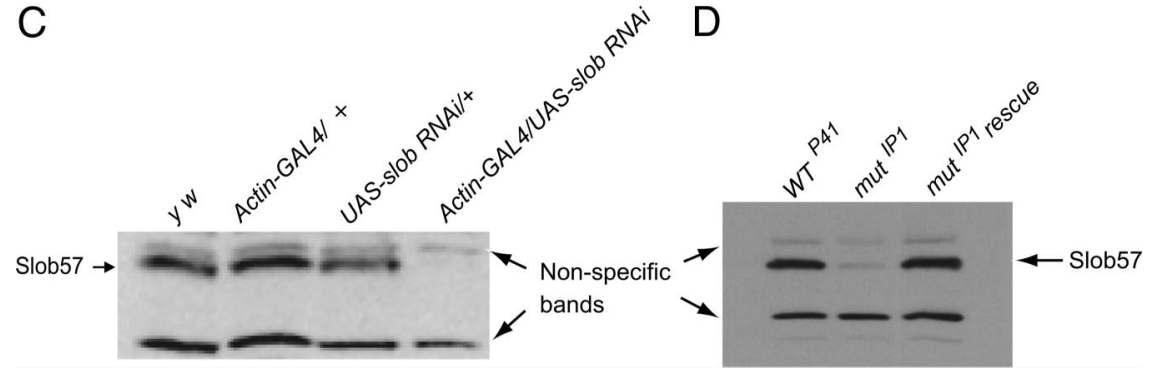

Figure 1. Molecular characterization of slob knock-out, knockdown and rescue flies. A, RT-PCR was done using total RNA from adult fly heads. Lane 1, DNA size markers (M). Compared with the parental line Kurs58 (lane 2), slob transcript is not detectable in the mutant lines mut ${ }^{\mathrm{K} 162}$ (lane 3) and mut ${ }^{\mathrm{P} 1}$ (lane 4), but is present in the precise excision line $\mathrm{WT}^{\mathrm{P} 41}$ (lane 5). Predicted product size is $1.1 \mathrm{~kb}$. $\boldsymbol{B}$, Western blot analysis using lysates from adult fly heads collected th the same time of day and probed with polyclonal anti-Slob antibody. In contrast to line Kurs58 (lane 1), Slob57 protein is dramatically reduced/absent in the mutant lines mut ${ }^{\mathrm{K} 162}$ (lane 2) and mut ${ }^{\mathrm{P} 1}$ (lane 3), and is recovered to at least wild-type levels in the precise excision line WT ${ }^{\mathrm{P} 41}$ (lane 4). C, Knockdown of Slob expression using transgenic RNAi. Western blot analysis conducted as described for $\boldsymbol{B}$ shows RNAi-induced knockdown of Slob57 expression. In contrast to the yw (lane 1), heterozygous Actin-GAL4 alone (Actin-GAL4/+, lane 2), and UAS-slob RNAi alone (UAS-slob RNAi/+, lane 3) control lysates, Slob57 expression is markedly reduced in lysates from flies carrying both Actin-GAL4 and UAS-slob RNAi (Actin-GAL4/UAS-slob RNAi, lane 4). D, Slob57 expression is restored to at least wild-type levels in rescue flies. Western blot conducted as described for $\boldsymbol{B}$ reveals that, in contrast to the lack of Slob expression in mut ${ }^{\mathrm{IP}^{11}}$ (lane 2), Slob57 levels in rescue flies (mut ${ }^{\mathrm{IP} 1}$ rescue, lane 3 ) are comparable to those seen in wild-type controls (WT ${ }^{\mathrm{P41}}$, lane 1). Similar restoration of Slob57 expression is observed upon rescue of Slob-RNAi knockdown flies (data not shown).

tion with dSlo, exerts a profound influence on neuronal membrane excitability in vivo. Interestingly, Slob-null flies also exhibit prolonged lifespan under conditions of complete food deprivation, suggesting a novel role for Slob in feeding behavior.

\section{Materials and Methods}

Fly strains. Flies were cultured at $25^{\circ} \mathrm{C}$ on standard Drosophila medium, and were kept in 12:12 h light:dark cycles. Electrophysiological recordings were performed between zeitgeber time 6 (ZT6) and ZT10. Mai301, Kurs45, and Kurs 58 lines were provided by Gunter Korge (Free University of Berlin, Berlin, Germany), dilp2-GAL4,UAS-GFP line by Eric Rulifson (University of California, San Francisco, San Francisco, CA), Actin-GAL4 and $y$ w; Ki $\Delta 2-3$ lines by Amita Sehgal (University of Pennsylvania School of Medicine, Philadelphia, $\mathrm{PA}$ ), and the UAS-GFP line was obtained from the Bloomington Stock Center.

Transgenic flies. For the construction of UAS-slob57 transgenics, fulllength slob57 cDNA was cloned into the commercially available P-element transformation vector, $p U A S T$, and transgenic lines were generated using standard procedures (Rubin and Spradling, 1982). For transgenic RNAi, the second half of $57 \mathrm{kDa}$ slob cDNA containing base pairs 598 1304 was cloned into the Sym-pUAST vector as described (Giordano et al., 2002). The Sym- $p U A S T$ vector consists of two convergent arrays of GAL4dependent $U A S$ sequences that drive simultaneous transcription of both sense and antisense strands, and thereby trigger the RNAi machinery to silence the corresponding target gene in vivo. Multiple, independently transformed lines were generated for each transgene construct, and were mapped to individual chromosomes.

P-element mutagenesis and screening. The P-element in the stock Kurs58 was induced to hop via a cross to $y$ w; Ki $\Delta 2-3$ line (which supplies the transposase enzyme required for the P-element to hop in the genome). This was followed by a series of crosses, which eventually yielded fly strains with new P-element insertions. Fly lines with putative new insertions were pooled and screened by genomic PCR for insertions in the slob gene. At least two flies from each genotype were collected for a total of 15 different putative novel insertion lines, homogenized for genomic DNA preparation, followed by PCR. For PCR, a barrage of gene-specific primers (supplemental Table 1, available at www.jneurosci.org as supplemental material) corresponding to genomic sequences in slob was used in combination with a PTR2 primer (5'-GAC-GGG-ACC-ACC-TTA-TGT-TA-3') in the inverted terminal repeat (ITR) region of the P-element. In case of a positive pool, singlefly PCR was done to identify the fly line with the desired insertion. PCR products were then sequenced to confirm juxtaposition of the P-element sequence and slob. One such line, called mut ${ }^{\mathrm{K} 162}$, was used for subsequent experiments.

To generate precise and imprecise excision lines, mut ${ }^{\mathrm{K} 162}$ flies were crossed to a $\Delta 2-3$ transposase line to induce P-element excisions in the slob gene. The $\mathrm{F} 1$ progeny resulting from this cross were used in subsequent crosses to eventually yield progeny with precise and imprecise excisions in the slob gene. Excision events were scored by white-eye phenotype (loss of the $w^{+}$marker of the P-element insert) and putative precise and imprecise excisions from the slob gene were tested by genomic PCR. For PCR, combinations of the following gene-specific primers that face each other and are separated by the P-element were used: 95LP 5'-GGC-TAG-TTC-CTT-GTT-TCCTCG-T-3'; 96LP 5'-TGC-ACG-TTT-GCTTCT-AGG-TG-3'; 96RP 5'-GCT-GCA-GCACTA-TCC-ACA-AA-3'; 97aRP 5' -CGC-TGTGTT-GTG-TAG-AGT-GTC-A-3'. Homozygous precise excisions yielded a product identical in size to that from the wild-type flies, while homozygous imprecise excisions that remove the P-element and a piece of the flanking DNA yielded no PCR product. No PCR products were seen with imprecise excision lines that had internal deletions in the inserted P-element as well, presumably because the remaining $\mathrm{P}$-element is too large to allow for PCR amplification with flanking primers. The precise excisions were confirmed by sequencing RT-PCR products of the fulllength $s l o b$ transcript. In an effort to define the molecular aberration in the imprecise excisions, we further analyzed these excision lines by PCR. For this, we used pairs of gene-specific primers along the entire length of the slob gene (supplemental Table 2, available at www.jneurosci.org as supplemental material). Two of the imprecise excision lines yielded products of the right size (similar to wild-type controls) with primer pairs along the entire slob gene, with the exception of primers flanking the P-element insertion, perhaps because of an internal deletion in the inserted P-element (that includes the $w^{+}$marker). To confirm that the failure to yield PCR products with flanking primers is due to an internal deletion in the inserted P-element, these two fly lines were further analyzed by PCR using the P-element primer PTR2 in combination with the following genespecific primers in the vicinity of the P-element insertion: 95LP $5^{\prime}$-GGC-TAGTTC-CTT-GTT-TCC-TCG-T-3'; 95RP 5'-ACG-AGG-AAA-CAA-GGAACT-AGC-A-3'; 97LP 5'-CTA-CCC-TGC-CGT-ATA-TTG-ATG-G-3'; 97bRP 5'-ACA-GCT-ATT-GAT-AGG-GCC-ACA-G-3'.

Genomic DNA preparation and PCR. A total of $\sim 30$ flies were homogenized in a buffer containing $7 \mathrm{~m}$ urea, 2\% SDS, 50 mм Tris, pH 7.5, 10 mM EDTA, pH 8.0, and $0.35 \mathrm{M} \mathrm{NaCl}$. DNA was extracted using phenol chloroform extraction followed by ethanol precipitation and resuspension in $30 \mu \mathrm{l}$ of water. PCRs were set up using $1 \mu \mathrm{l}$ of genomic DNA as template, and the inverted-repeat primer PTR2 in combination with a series of 40 primers (supplemental Table 1, available at www.jneurosci. org as supplemental material) spanning the entire length of the slob gene. All PCRs were conducted in a $50 \mu \mathrm{l}$ reaction volume with TaqDNA polymerase (Promega) and the following PCR program: $95^{\circ} \mathrm{C}$ for $5 \mathrm{~min}$; 35 cycles of $95^{\circ} \mathrm{C}$ for $30 \mathrm{~s}, 60^{\circ} \mathrm{C}$ for $1 \mathrm{~min}, 68^{\circ} \mathrm{C}$ for $5 \mathrm{~min} ; 72^{\circ} \mathrm{C}$ for $10 \mathrm{~min}$. 
Single-fly PCR. Each fly was homogenized for $\sim 30 \mathrm{~s}$ in $50 \mu \mathrm{l}$ of a buffer containing $10 \mathrm{~mm}$ Tris-Cl, pH 8.2, 1 mm EDTA, 25 mm NaCl, and $200 \mu \mathrm{g} / \mathrm{ml}$ proteinase $\mathrm{K}$. The fly extract was then incubated at $25^{\circ} \mathrm{C}$ for $\sim 30 \mathrm{~min}$, followed by heating at $95^{\circ} \mathrm{C}$ for $10 \mathrm{~min}$ to inactivate proteinase K. For PCR, $5 \mu \mathrm{l}$ of this prep was used as template with slob gene-specific primers in combination with the inverted-repeat primer PTR2. PCRs were conducted using the same program as described above in Genomic DNA preparation and PCR, and PCR products were sequenced to confirm identity.

$R T-P C R$. Total RNA was extracted from $\sim 50$ fly heads using Ultraspec RNA isolation System following the manufacturer's recommendations (Biotecx Laboratories). Approximately $5 \mu \mathrm{g}$ of total RNA was reverse transcribed with $50 \mathrm{ng}$ random hexamers using the Superscript First strand Synthesis System (Invitrogen). For PCR, 10\% of the first strand reaction was used as template with $5^{\prime}$-CTC AGG AAT GGA TTC AAG GTG-3' forward primer and 5'-GGT TCG GGT TCG GAT TTC TT-3' reverse primer. The following PCR program was used: $94^{\circ} \mathrm{C}$ for $3 \mathrm{~min} ; 35$ cycles of $94^{\circ} \mathrm{C}$ for $30 \mathrm{~s}, 60^{\circ} \mathrm{C}$ for $1 \mathrm{~min}, 68^{\circ} \mathrm{C}$ for $2 \mathrm{~min}$; $72^{\circ} \mathrm{C}$ for $10 \mathrm{~min}$. PCR products were separated on a $1 \%$ agarose gel and viewed using Kodak Image Station 4000R. The $1 \mathrm{~kb}$ ladder from New England Biolabs was used as the molecular weight standard.

Slob antibody purification. Polyclonal Slob antibody was purified as described previously using serum from rabbits immunized with a GSTSlob fusion protein (Jaramillo et al., 2004).

Western blots. Fly heads collected at the same time of day were homogenized in a lysis buffer containing 1\% CHAPS, 20 mm Tris- $\mathrm{HCl} \mathrm{pH}$ 7.5, 10 mм EDTA, $120 \mathrm{~mm} \mathrm{NaCl}, 50 \mathrm{~mm} \mathrm{KCl}, 2$ $\mathrm{mM}$ DTT, and protease inhibitors $[1 \mathrm{~mm}$ PMSF, $1 \mu \mathrm{g} / \mu \mathrm{l}$ each aprotinin, leupeptin, and pepstatin A (Sigma) ]. Protein concentration in the lysates was determined using the Bio-Rad DC Protein Assay. Equal concentrations of protein were loaded on a polyacrylamide gel, and transferred onto nitrocellulose membranes. Blots were blocked with $5 \%$ nonfat milk in

TBST ( $0.1 \%$ Tween 20 in Tris-buffered saline) and probed with anti-Slob antibody overnight. The following day, blots were washed with TBST, incubated with horseradish peroxidase-conjugated donkey anti-rabbit secondary antibody (GE Healthcare) for $\sim 1 \mathrm{~h}$, and washed again with TBST, and the proteins were detected using Enhanced Chemiluminescence Detection System (GE Healthcare).

Fly crosses for electrophysiology. For transgenic expression of Slob57 in PI-1 neurons in a wild-type genetic background, UAS-slob57 flies were crossed to dilp2-GAL4, UAS-GFP flies, and the resulting progeny (dilp2GAL4,UAS-GFP/+; UAS-slob57/+) were used for electrophysiology. For electrophysiological analysis of slob mutants, Mai301 GAL4 and UAS-GFP flies were crossed into a homozygous slob mutant (mut ${ }^{\mathrm{IP} 1}$ ) background. $m u t^{I P 1} ;$ Mai301 flies were then crossed to mut ${ }^{\mathrm{IP1}} ;$ UAS-GFP flies to achieve GFP expression in the PI-1 neurons of slob mutants. Transgenic rescue of the physiological phenotype observed in slob mutants was accomplished by the following crosses. UAS-GFP and $m u t^{I P 1}$ were recombined on the second chromosome, and Mai301 GAL4 and UAS-slob57 were recombined on the third chromosome. The recombinants, mut ${ }^{\mathrm{IP1}}$, UAS-GFP were then crossed to Mai301 GAL4,UAS-slob57, and the resulting heterozygous progeny were then intercrossed to obtain mut ${ }^{\mathrm{IP} 1}$, UAS-GFP; Mai301 GAL4,UAS-slob57 for patch-clamp recordings.
For electrophysiological analysis of transgenic slob RNAi flies, UASslob RNAi;Mai301 GAL4 flies were crossed to UAS-GFP flies to target GFP and slob RNAi to the PI-1 neurons (among other neurons). UASslob RNAi/UAS-GFP;Mai301 GAL4/+ flies that have one copy of each of the transgenes were then used for patch recording. To rescue slob RNAi flies, UAS-slob RNAi;Mai301 GAL4 flies were crossed to UAS-GFP; UASslob57 flies, and the resulting heterozygous progeny (UAS-slob RNAi/ UAS-GFP;Mai301 GAL4/UAS-slob57) containing one copy each of the transgenes was subjected to electrophysiology.

In vivo electrophysiology. For in vivo patch recording from PI neurons, flies were anesthetized with $\mathrm{CO}_{2}$ and glued ventral side down to a coverslip. The cuticle was peeled off to expose the surface of the brain, and the coverslip was placed in a chamber containing extracellular solution ( $\mathrm{NaCl} 130, \mathrm{KCl} 5, \mathrm{MgCl}_{2} 2, \mathrm{CaCl}_{2}$ 2, sucrose 30, HEPES $10 \mathrm{~mm}$ with $\mathrm{pH}$ 7.2). The chamber was placed on the stage of an Olympus BX51 fluorescent microscope, and PI neurons were identified by their location and bright green fluorescence. Patch recording electrodes (WPI) were fire polished, and had resistances from 4 to $6 \mathrm{M} \Omega$ when filled with intracellular solution ( $\mathrm{KCl} 140, \mathrm{MgCl}_{2} 1, \mathrm{CaCl}_{2}$ 2, EGTA 5, HEPES $10 \mathrm{~mm}$ with $\mathrm{pH}$ 7.2). Standard techniques were used to record single-channel currents in the cell-attached recording mode, macroscopic currents in the 

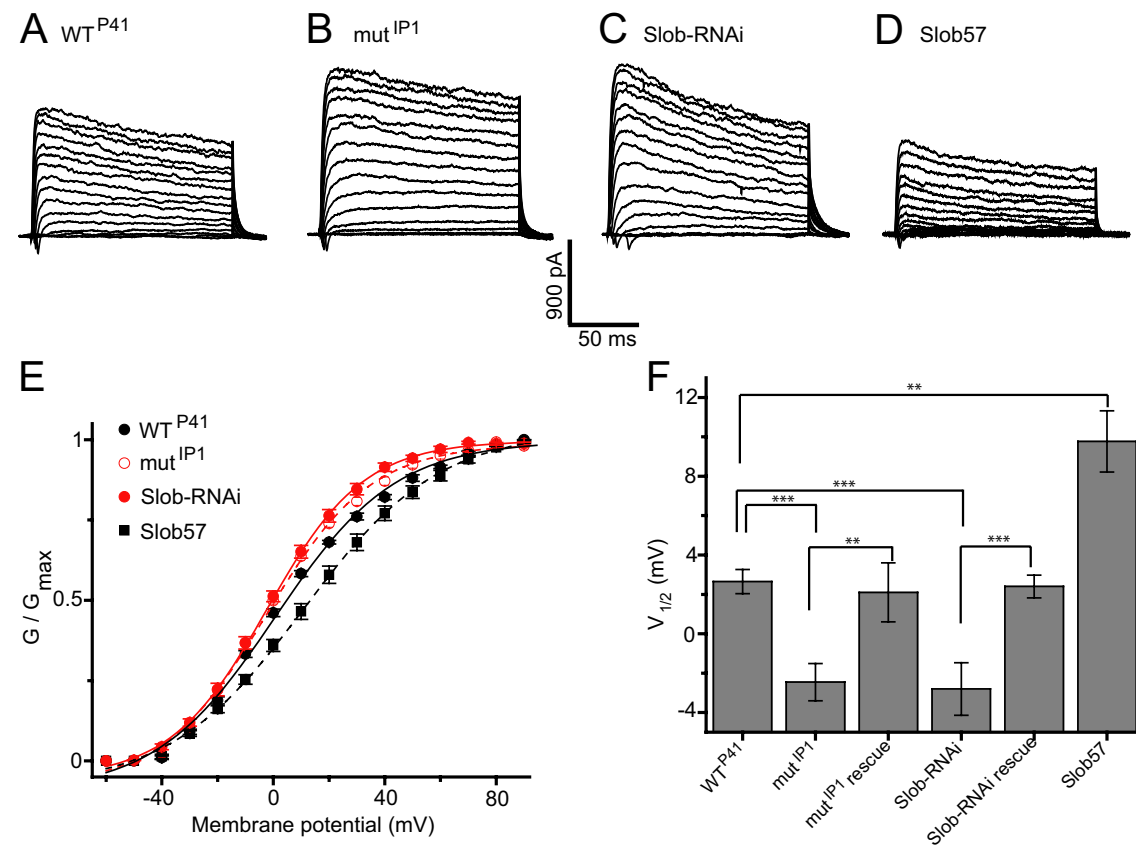

E

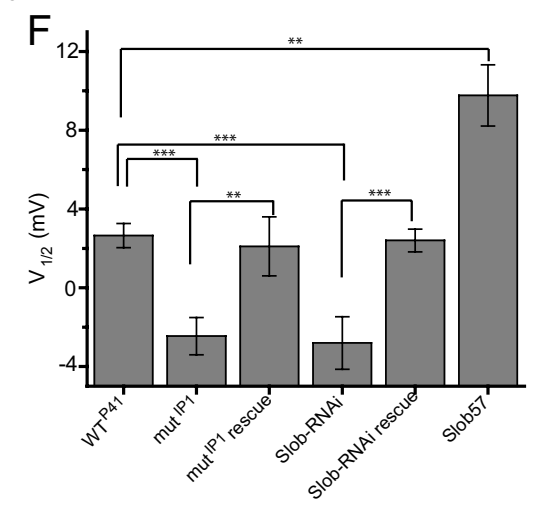

Figure 3. Whole-cell patch recording reveals the modulation of outward current by endogenous Slob in PI-1 neurons in vivo. Small inward and large outward currents were evoked by $150 \mathrm{~ms}$ depolarizing voltage steps from a holding potential of $-70 \mathrm{mV}$, to pulse potentials ranging from -60 to $+90 \mathrm{mV}$ in $10 \mathrm{mV}$ increments. Currents from the following fly genotypes are shown: $\mathrm{WT}^{\mathrm{P41}}(\boldsymbol{A})$, mut ${ }^{\mathrm{IP} 1}(\boldsymbol{B})$, transgenic Slob-RNAi (expression driven by Mai301-GAL4) (C), and transgenic Slob57 (expression driven by dilp2-GAL4 in a wild-type background) (D). $\boldsymbol{E}$, Conductance-voltage relationships for the peak outward currents shown in $\boldsymbol{A}-\boldsymbol{D}$. Data are shown as mean \pm SEM, and the lines are fits to the Boltzmann equation. $\boldsymbol{F}$, The voltage required for half-maximal activation of the outward current $\left(V_{1 / 2}\right)$ is shown for recordings from PI-1 neurons of various fly genotypes (mean \pm SEM). Differences between pairs of genotypes are significantly different as shown $\left(n=10-14\right.$; ${ }^{* *} p<0.01$; $\left.{ }^{* * *} p<0.001\right)$.
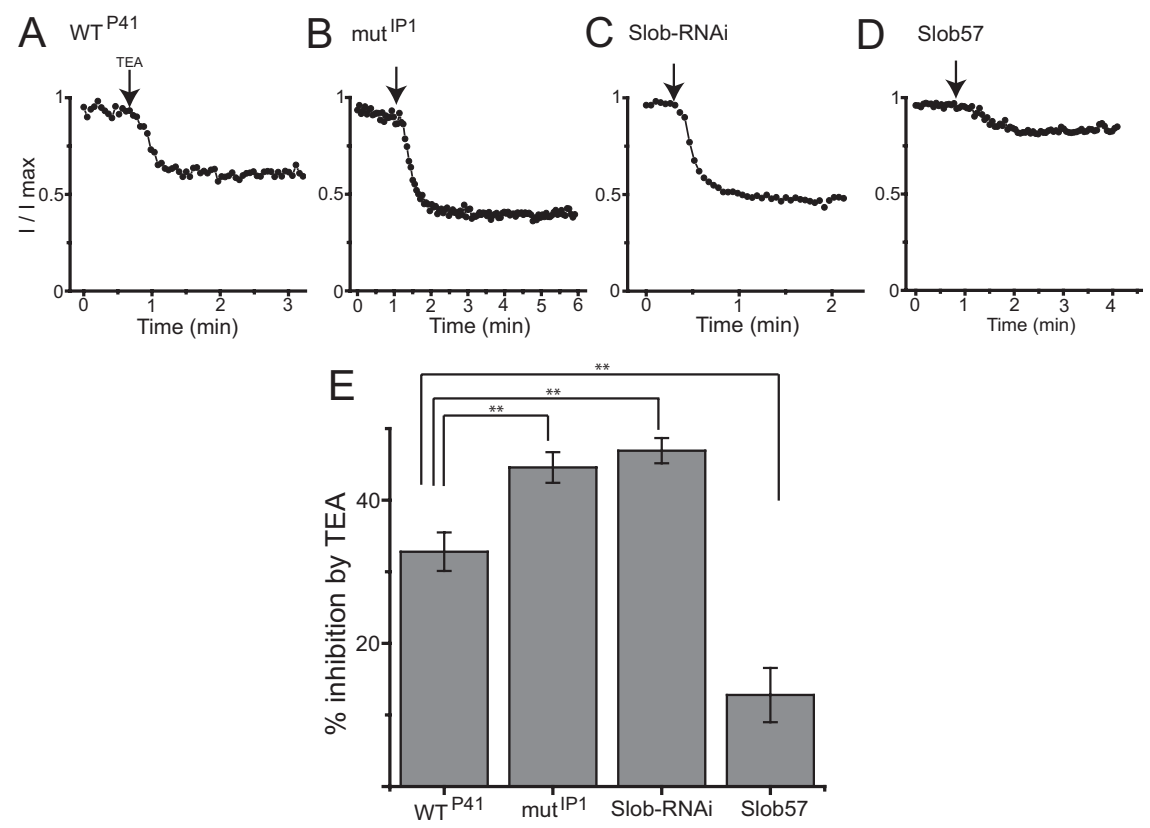

Figure 4. The proportion of the total neuronal outward current that is dependent on the dSlo-specific channel blocker TEA is dependent on the Slob genotype. Outward currents were evoked by repetitive voltage steps from a holding potential of $-70 \mathrm{mV}$ to a pulse potential of $+60 \mathrm{mV}$, and $1 \mathrm{~mm}$ TEA was added to the bath solution at the times indicated by the vertical arrows. Normalized peak amplitude of the outward current $\left(I / I_{\max }\right)$ is plotted as a function of time from the start of recording, for the following fly genotypes: $\mathrm{WT}^{\mathrm{P41}}(\boldsymbol{A})$, mut ${ }^{\mathrm{P1}}(\boldsymbol{B})$, transgenic Slob-RNAi (expression driven by Mai301-GAL4) $(\boldsymbol{C})$, and transgenic Slob57 (expression driven by dilp2-GAL4 in a wild-type background) (D). $\boldsymbol{E}$, The proportion of neuronal outward current inhibited by $1 \mathrm{~mm}$ TEA differs significantly between fly genotypes $\left(n=9-11 ;{ }^{* *} p<0.01\right)$. whole-cell voltage-clamp mode, and voltage in current-clamp mode, with an Axopatch 200A amplifier (Molecular Devices). Data were digitized with a Digidata 1322A interface (Molecular Devices) and stored on a PC hard drive for further analysis with pClamp9 software (Molecular Devices). The $n$ value given for each experiment refers to the number of individual neurons analyzed. Grouped data are presented as mean \pm SEM. Statistical comparisons between groups were performed by one-way ANOVA, and the level of significance is indicated by asterisks as shown.

Single-cell RT-PCR. dilp2-GAL4,UAS-GFP and Kurs45/+;UAS-GFP/+ flies were used to visualize PI-1 and PI-3 neurons, respectively. Patch electrodes were used to aspirate individual identified PI neurons from flies that were prepared for electrophysiological recordings. RNase inhibitor solution was prepared using

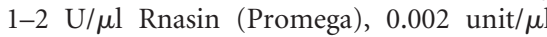
RQ1 RNase-free DNase (Promega), and $1 \mu \mathrm{M}$ dithiothreitol. Once a cell was identified based both on location and GFP fluorescence under an Olympus BX51WI upright microscope, a pipette filled with intracellular saline was positioned close to the cell. After a seal was obtained and whole-cell patch mode was achieved, suction was applied to draw the cytoplasm slowly into the tip of the pipette. The pipette was immediately transferred to an RNase-free $0.2 \mathrm{ml}$ PCR tube containing $10 \mu \mathrm{l}$ of RNase-free solution, and its tip was broken to allow the contents to enter the tube. The tube was frozen on an ethyl alcohol/dry ice bath. The contents of each tube were centrifuged using a bench-top centrifuge at maximum speed for $30 \mathrm{~s}$. All samples were first incubated at $94^{\circ} \mathrm{C}$ for $10 \mathrm{~min}$, and reagents were then added. For both RTPCR and PCR, the following forward primer was used: 5'-GGA-TCG-ACA-ATC-GGA-ACAGT-3'. The reverse primers used for RT-PCR and PCR were 5' -CAC-ATT-GGC-GCT-TCT-TTACC- $3^{\prime}$ and $5^{\prime}$-GAA-GAG-CAG-TGC-CTCCAG-AA-3', respectively. Superscript Onestep RT-PCR with Platinum Taq (Invitrogen) was used for the RT-PCR, and Taq polymerase (Invitrogen) for the subsequent PCR. PCR products were separated on a $1 \%$ agarose gel and viewed using Kodak Image Station 4000R. The $1 \mathrm{~kb}$ ladder from New England Biolabs was used as the molecular weight standard. Products were sequenced to confirm identity.

Starvation assay. This was performed as described previously (Sarov-Blat et al., 2000) with slight modifications. Flies $<24$ h old were aged for an additional $3 \mathrm{~d}$ in an LD cycle. At ZT11, flies were transferred individually to tubes containing $1.5 \%$ agar without food, and monitored for activity using the Trikinetics activity monitoring system. Over the subsequent $4 \mathrm{~d}$, average activity and the fraction of active flies (reflecting survival) were monitored as a function of starvation duration in LD. The log-rank test was used for statistical comparison between different fly strains in each dataset (Lawless, 1982). For activity plots under starvation conditions, activity events in $30 \mathrm{~min}$ intervals were averaged for the entire population of flies tested for each genotype. 


\section{Results}

Isolation of slob-null alleles and Slob knockdown flies

Using the fly stock Kurs58 (Siegmund and Korge, 2001) that has a P-element insertion $\sim 27 \mathrm{~kb}$ upstream from the slob gene, we used a local hopping strategy combined with PCR-based screening to recover a P-element insertion in the slob gene. Among 200 dysgenic lines screened for an insertion in the slob gene, one line, called mut ${ }^{\mathrm{K} 162}$, had a P-element located in a $3^{\prime}$ exon of the gene. The P-element in mut ${ }^{\mathrm{K} 162}$ was then remobilized to generate several precise excision control lines and 14 imprecise excision mutant lines. The precise excision line, $\mathrm{WT}^{\mathrm{P} 41}$ was confirmed by sequencing the PCR products of slob transcripts. Two of the fourteen imprecise excision lines, mut ${ }^{\mathrm{IP} 1}$ and mut ${ }^{\mathrm{IP} 2}$ have internal deletions in the P-element that is inserted in the slob gene. For all other imprecise excision lines, no PCR products were detected with a series of slob-specific primers, suggesting that the excision lines have deletions in the slob gene. Unfortunately, the deletions in all the latter mutant lines run into neighboring genes. Since mut $^{\mathrm{K} 162}$, mut ${ }^{\mathrm{IP} 1}$, and mut ${ }^{\mathrm{IP} 2}$ are the cleanest of the mutant lines, we used two of these lines, mut ${ }^{\mathrm{K} 162}$ and mut ${ }^{\mathrm{IP} 1}$, for further molecular and phenotypic characterization. The precise excision line, $\mathrm{WT}^{\mathrm{P} 41}$, served as the wild-type control.

Using RT-PCR with PCR primers that are predicted to amplify the 3 ' end of all slob transcripts, we found that full-length slob transcripts are not detectable in the P-element insertion line, mut $^{\mathrm{K} 162}$, or in the imprecise excision line mut ${ }^{\mathrm{IP} 1}$, while they are present in $\mathrm{WT}^{\mathrm{P} 41}$ (Fig. 1A). Full-length transcripts are not detectable for the remaining imprecise excision lines as well (data not shown). In addition, Western blots using a polyclonal antiSlob antibody demonstrate that the major Slob isoform, the 57 $\mathrm{kDa}$ Slob57 protein, is significantly reduced or absent in all slob mutants, whereas it is restored to at least wild-type levels in the precise excision line $\mathrm{WT}^{\mathrm{P} 41}$ (Fig. $1 B$ ). A weak Western blot signal with the same molecular weight as Slob57 remains in all mutants, regardless of the type of molecular aberration, suggesting that it may be a cross-reacting band but not residual Slob protein. Furthermore, the residual signal is not detectable after immunoprecipitation with anti-Slob antibody, confirming that it is likely to be a cross-reacting band (data not shown). Finally, we find that no new protein bands appear on Western blots from slob mutant flies, eliminating the possibility that the mutants are making truncated Slob protein(s). Although the anti-Slob antibody generated in our lab recognizes only Slob57 on fly head Western blots, the exons affected by the P-element mutagenesis are represented in all slob transcripts. Furthermore, because none of the transcripts are detectable by RT-PCR analysis of slob mutants, it is likely that these mutants lack all Slobs.

We also used transgenic RNAi to construct Slob knockdown flies. As shown in Figure 1C, UAS-slob RNAi, when driven by the ubiquitous Actin-GAL4 driver, knocks down Slob57 protein expression. Because the slob cDNA used to produce the RNAi flies contains the slob sequence that is common to all slob transcripts, it is very likely that all Slob protein isoforms are knocked down in these flies. Finally, we constructed rescue flies by transgenic expression of Slob57, under the control of the Actin-GAL4 driver, in the mut ${ }^{\mathrm{IP1}}$ and Slob-RNAi genetic backgrounds. In both of these fly lines, rescue restores the expression of Slob57 to at least that seen in $\mathrm{WT}^{\mathrm{P} 41}$ flies (Fig. $1 \mathrm{D}$ and data not shown).

\section{Slob expression level influences outward current recorded from PI neurons in vivo}

To facilitate whole-cell and single-channel recordings from PI neurons in the brains of living flies, we constructed flies in which
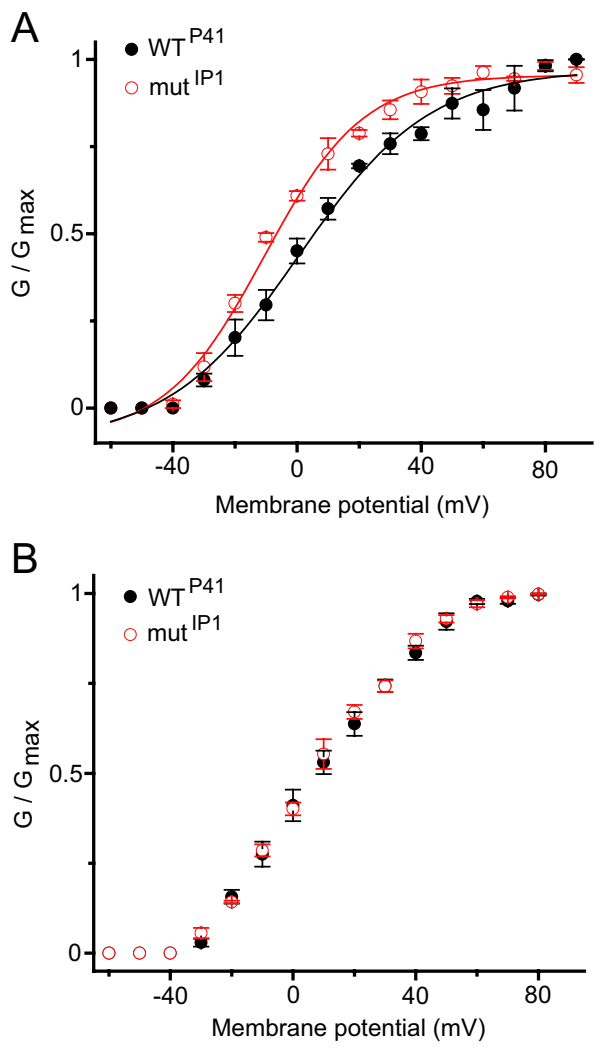

Figure 5. Conductance-voltage relationships of the TEA-sensitive and TEA-insensitive components of the outward current in PI neurons. The protocol was as for Figure 3. Peak outward currents following application of $1 \mathrm{~mm}$ TEA were subtracted from the currents before adding TEA, and the conductance-voltage relationships were computed from the subtracted traces. $\boldsymbol{A}$, TEA-sensitive component of conductance, attributable to dSIo. $\boldsymbol{B}$, TEA-insensitive component of conductance, attributable to all outward current components other than dSlo. Data are shown as mean \pm SEM, and the lines are fits to the Boltzmann equation.

the PI neurons were visualized by driving the expression of green fluorescent protein (GFP) under the control of the Kurs45-, dilp2-, or Mai301-GAL4 drivers. Flies were immobilized by gluing them ventral side down on a coverslip (Fig. $2 A$ ), and the cuticle was peeled back carefully to expose the surface of the brain (Fig. 2B). The GFP-expressing PI neurons were visualized by illumination with a fluorescent source (Fig. 2C,D), and a patch recording electrode (Fig. $2 D$ ) was used to obtain gigaseal recordings from the green neurons, in either the cell-attached or wholecell recording modes. To confirm that these neurons do indeed express Slob, single-cell RT-PCR was used to amplify Slob mRNA in the recording electrode following whole-cell recording. As shown in Figure $2 E$, Slob message is detected in at least two of the three previously defined subsets of PI neurons, PI-1 and PI-3.

We examined currents evoked by depolarizing voltage steps in the whole-cell recording mode. Fast inward currents probably carried by sodium ions were seen occasionally, and robust outward voltage-dependent potassium currents were observed routinely (Fig. 3A). No differences were observed between PI-1 (DILP-secreting) and PI-3 neurons in the voltage dependence of the outward current (data not shown). Accordingly all experiments shown here were performed with PI-1 neurons, visualized with either the dilp2- or Mai301-GAL4 drivers. The total voltagedependent outward current arises from the multiple potassium channels, including dSlo, that are expressed in Drosophila neurons and muscle. To determine whether Slob influences this total outward current in vivo, we recorded from PI-1 neurons in flies in 

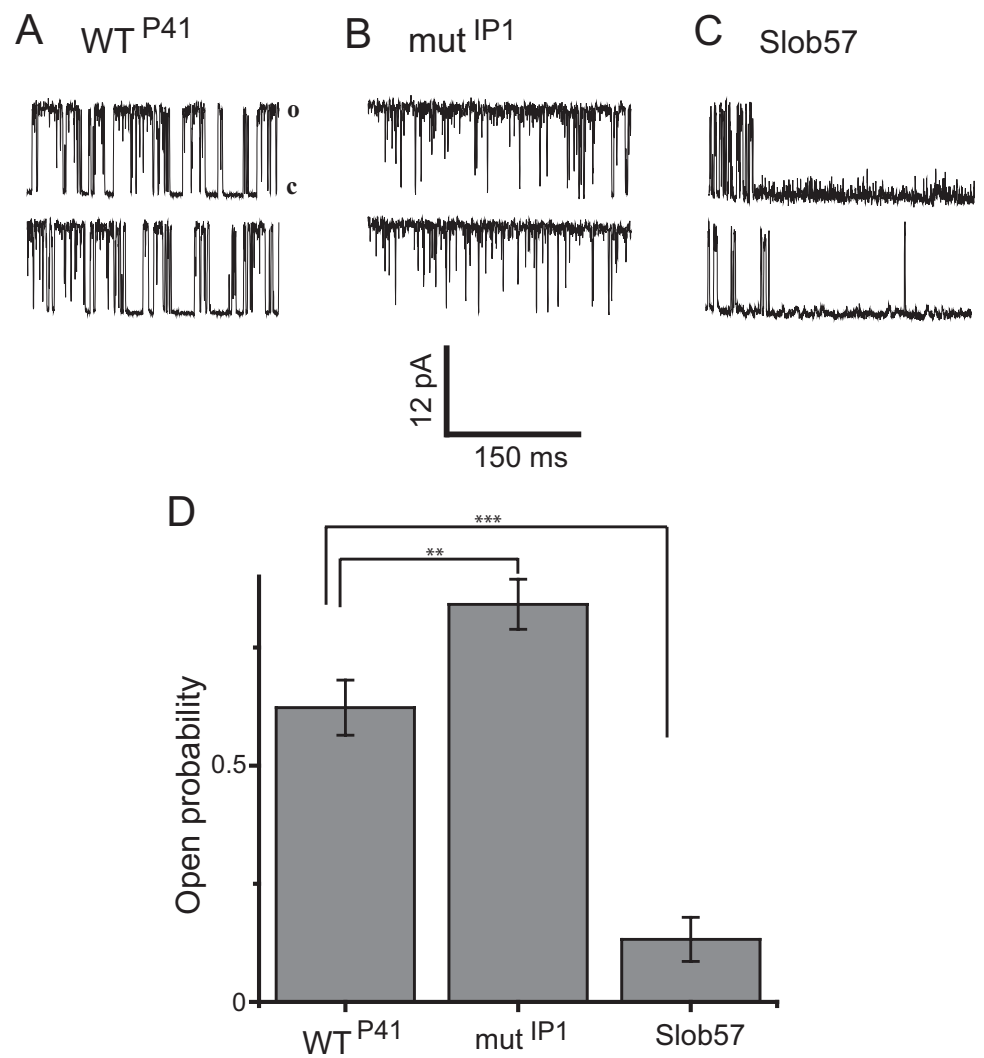

Figure 6. The gating of single dSlo channels in cell-attached patches on PI-1 neurons in vivo is dependent on the Slob genotype. Single-channel currents were evoked by prolonged step depolarizations from a holding potential of $-70 \mathrm{mV}$ to a pulse potential of $+60 \mathrm{mV}$, using flies of the following genotypes: $\mathrm{WT}^{\mathrm{P} 41}(\boldsymbol{A})$, mut ${ }^{\mathrm{P1}}(\boldsymbol{B})$, and transgenic Slob57 (in a wild-type background) (C). $D$, The dSlo channel open probability during at least 1 min of continuous recording differs significantly between fly genotypes as shown $\left(n=4-12 ;{ }^{* *} p<0.01 ;{ }^{* * *} p<0.001\right)$.

which Slob expression was eliminated by P-element mutagenesis. As shown in Figure $3 B$, the outward current traces evoked by each depolarizing voltage step are larger in the absence than in the presence of Slob (compare with Fig. $3 A$ ). Quantitative analysis reveals that the voltage dependence of activation, as measured by the conductance versus voltage $(G-V)$ relationship, is shifted in the hyperpolarizing direction in the Slob-null flies (Fig. $3 E, F$ ). A similar increase in outward current (Fig. $3 C$ ) and hyperpolarizing shift in the $G-V$ relationship (Fig. $3 E, F$ ) is observed in PI-1 neurons in which Slob expression is attenuated by the targeted expression of transgenic Slob-RNAi under the control of the Mai301 promoter. The shifts in the voltage at which halfmaximal activation of the outward current occurs $\left(V_{1 / 2}\right)$ are modest, but highly significant (Fig. $3 F$ ).

These similar findings using two completely independent methods of decreasing Slob expression suggest strongly that the changes in the voltage dependence of dSlo activation result from the manipulation of Slob, rather than any experimental artifact of either P-element mutagenesis or RNAi expression. This is confirmed by the finding that the outward current phenotype observed in the Slob knock-out/knockdown flies can be rescued by the transgenic expression of Slob57 in PI-1 neurons, in either the mut $^{\mathrm{IP} 1}$ Slob-null or Slob-RNAi genetic background (Fig. $3 F$ ). Note also that the $G-V$ curve is shifted in the depolarizing direction when Slob57 is expressed transgenically in the PI-1 neurons in wild-type flies that express normal endogenous levels of Slob (Fig. 3D-F). Interestingly, in this case the change in $V_{1 / 2}$ is larger than that elicited by Slob knock-out/knockdown (Fig. $3 F$ ), sug- gesting that only a fraction of PI-1 neuron dSlo channels are bound to Slob in wildtype flies.

\section{Slob selectively modulates a TEA- sensitive component of the outward current}

To determine whether these effects of Slob on the total outward current are attributable to its modulation of dSlo, we made use of the potassium channel blocker tetraethylammonium (TEA), which at concentrations at or below $1 \mathrm{~mm}$ selectively blocks dSlo and has minimal effect on other Drosophila potassium channels (Shen et al., 1994). As shown in Figure 4, A and $E$, application of $1 \mathrm{~mm}$ TEA to the bathing solution blocks $\sim 33 \%$ of the total outward current in PI-1 neurons in wildtype flies. The proportion of outward current blocked by TEA increases to $\sim 45 \%$ in mut ${ }^{\mathrm{IP1}}$ Slob-null flies (Fig. $4 B, E$ ) or SlobRNAi knockdown flies (Fig. 4C,E), consistent with the idea that dSlo current is selectively increased in the absence of Slob. In contrast, the proportion of the outward current subject to TEA block is only $\sim 15 \%$ when transgenic Slob57 is expressed in the PI-1 neurons in a wild-type genetic background (Fig. 4D,E). These findings confirm that the proportion of PI-1 neuronal outward current attributable to dSlo in vivo is dependent on the level of expression of Slob in those neurons.

We also examined separately the $G-V$ relationships of the TEA-sensitive and TEA-insensitive components of the outward current. As shown in Figure 5A, Slob deletion shifts the $G-V$ relationship of the TEA-sensitive component of outward current, attributable to dSlo, in the hyperpolarizing direction; this shift is greater than the $G-V$ shift of the total outward current (compare with Fig. $3 E$ ). In contrast, the $G-V$ relationship of the TEAinsensitive component of the current, comprising all neuronal potassium current other than that attributable to dSlo, is not influenced at all by Slob deletion (Fig. 5B). These findings confirm further that dSlo is the predominant target of Slob, and suggest also that other neuronal potassium channels are not modulated by Slob.

\section{In vivo single-channel recording from PI neurons}

We used cell-attached patch single-channel recordings to assess more directly the effects of Slob on neuronal dSlo channel activity in vivo. Because the single-channel conductance of dSlo is $\sim 180$ pS, at least fivefold greater than that of any other Drosophila potassium channel, dSlo can be identified unequivocally in single-channel recordings. In PI-1 neurons in the brains of wildtype flies, we chose recording conditions under which individual dSlo channels open and close rapidly and exhibit an open probability $\left(P_{\mathrm{o}}\right)$ of $\sim 0.5$ (Fig. $6 A$ ). When the same conditions were used to record single dSlo channels in PI-1 neurons in mut ${ }^{\text {IP1 }}$ Slob-null flies, we found that the channels are open virtually all the time, closing only occasionally and briefly (Fig. $6 \mathrm{~B}$ ). In contrast, in PI-1 neurons in the brains of flies in which transgenic 
A
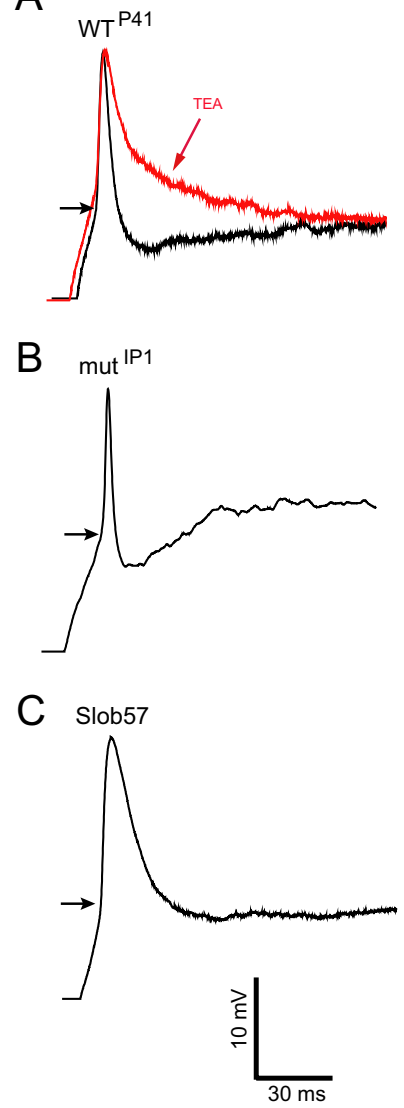

Figure 7. Action potential duration in PI-1 neurons is dependent on the Slob genotype. In all cases, action potentials were evoked under whole-cell current clamp. The starting voltage was adjusted to $-80 \mathrm{mV}$ by the injection of an appropriate amount of holding current, and action potentials were evoked by the subsequent injection of $100 \mathrm{pA}$ of depolarizing current. The duration of the action potential was measured at the inflection point of the depolarizing phase, shown by the horizontal arrows in $\boldsymbol{A}-\boldsymbol{C}$. $\boldsymbol{A}$, The duration of the action potential in WT ${ }^{\mathrm{P41}}$ flies is increased by $1 \mathrm{~mm}$ TEA, confirming a role for dSlo in action potential repolarization. Action potential duration is shorter in mut ${ }^{\mathrm{P} 1}$ flies $(\boldsymbol{B}, \boldsymbol{D})$, and is longer in flies expressing transgenic Slob57 $(\boldsymbol{C}, \boldsymbol{D})$. $\boldsymbol{E}$, The repolarizing phase of the action potential was fit with a single exponential function, and the extracted mean time constants of repolarization \pm SEM are shown for the different fly genotypes. Differences between genotypes in action potential width $(\boldsymbol{D})$ and repolarization time constant $(\boldsymbol{E})$ are significant as shown $\left(n=3-5\right.$; $\left.{ }^{* *} p<0.01 ;{ }^{* * *} p<0.001\right)$.

Slob57 was expressed under the control of the dilp2 promoter, dSlo channels spend most of their time closed, opening only occasionally and briefly (Fig. 6C). The data from multiple recordings are quantified in Figure $6 D$, which demonstrates that dSlo channel open probability is modulated profoundly in vivo by the Slob genotype. Kinetic analysis of the single-channel records (data not shown) confirms what is evident from visual inspection of Figure $6 A-C$, that the predominant action of Slob is to increase the dwell time of dSlo channels in a closed state or states.

\section{Modulation of action potentials in PI neurons}

We also asked whether Slob expression level influences the properties of action potentials in the PI-1 neurons. These neurons do not fire action potentials spontaneously in vivo under our wholecell recording conditions, but we were able to elicit action potentials with depolarizing current injections in the current-clamp recording mode. As can be seen in Figure $7 A$, action potential duration in PI-1 neurons is increased by $1 \mathrm{~mm}$ TEA, consistent with the idea that large-conductance calcium-activated potassium channels activate rapidly during the action potential and thereby contribute significantly to its repolarizing phase (Vergara et al., 1998; Shao et al., 1999). Action potential duration is shorter in PI-1 neurons in the brains of Slob-null flies than in those in wild-type flies (Fig. $7 \mathrm{~B}, \mathrm{D}$ ). This is due to an increase in the rate of repolarization (Fig. $7 E$ ), whereas the rise times of the action potentials are similar in the two genotypes. These findings are consistent with the hypothesis that knocking down Slob in vivo will disinhibit dSlo, thereby leading to an increase in the rate of action potential repolarization. In contrast, action potential duration is increased in PI-1 neurons in flies in which transgenic Slob57 is expressed under the control of the dilp2 promoter (Fig. $7 C, D$ ), again as a result of a change in the rate of repolarization (Fig. $7 E$ ). As is the case for the shift in the $G-V$ relationship (Fig. $3 F$ ), the effect of transgenic expression of Slob57 on action potential duration and rate of repolarization is greater than that of knocking out Slob expression (Fig. $7 D, E$ ).

\section{Slob mutant flies show an increase in starvation resistance}

Clearly Slob regulates the membrane excitability of PI neurons in vivo. To explore the downstream effects of such modulation on fly behavior, we tested Slob-null mutants in two behaviors in which the PI neurons participate in a variety of insect species (Sokolove and Loher, 1975; Zaretsky and Loher, 1983; Krauthamer, 1985; Kaneko and Hall, 2000). In particular, we hypothesized that Slob, by modulating the excitability of PI neurons, regulates circadian rhythms and/or energy metabolism/feeding behavior. Since a variety of recent studies suggest a role for slob in circadian rhythms (Claridge-Chang et al., 2001; McDonald and Rosbash, 2001; Ceriani et al., 2002; Lin et al., 2002; Ueda et al., 2002; Jaramillo et al., 2004), we first tested slob mutants in several circadian behavioral assays. Slob-null flies show no obvious phenotype in two classical circadian behavioral assays, locomotor activity rhythms and eclosion rhythms. Furthermore, circadian oscillations in the levels of the clock proteins PERIOD (PER) and TIMELESS (TIM) in fly heads are not affected by Slob deletion, nor is the oscillation of luciferase reporter expression in whole flies, driven by the per promoter (data not shown). To ask whether Slob participates in the regulation of feeding behavior, we tested slob mutants in a starvation resistance assay, wherein we monitored the lifespan of individual flies under conditions of complete food deprivation. Interestingly, the results indicate that slob mutant flies survive starvation significantly longer than do wild-type control flies (Fig. 8A). Half-lives of homozygous mut ${ }^{\mathrm{K} 162}$, mut ${ }^{\mathrm{IP} 1}$, and the transheterozygous mut ${ }^{\mathrm{K} 162} /$ mut $^{\mathrm{IP} 1}$ flies are $33-48 \%$ longer than those of $\mathrm{WT}^{\mathrm{P} 41}$ flies (Table 1). A similar phenotype was reported previously for flies with decreased insulin signaling (Clancy et al., 2001; Kramer et al., 2003) and corpora cardiaca (CC)-ablated flies (Lee and Park, 2004).

To test the possibility that the starvation phenotype arises from a second site mutation in the genetic background rather than the lack of Slob, we conducted a deficiency mapping experiment. As shown in Figure $8 B$, similar to Slob-null mutants, both mut ${ }^{\mathrm{K} 162} / \mathrm{Df}$ and mut ${ }^{\mathrm{IP} 1} / \mathrm{Df}$ flies survive significantly longer than do $\mathrm{WT}^{\mathrm{P} 41} / \mathrm{Df}$ and heterozygous $\mathrm{Df} /+$, mut ${ }^{\mathrm{K} 162} /+$, and mut ${ }^{\mathrm{IP} 1} /+$ control flies. Half-lives of mut ${ }^{\mathrm{K} 162} / \mathrm{Df}$ and mut ${ }^{\mathrm{IP} 1} / \mathrm{Df}$ flies are $30 \%$ and $32 \%$ longer, respectively, than the half-lives of the control groups (Table 1). These results indicate that the enhanced starvation resistance phenotype in Slob-null mutant flies maps close to the slob locus.

\section{Discussion}

Our results provide a direct demonstration that an auxiliary protein associated with a neuronal potassium channel can modulate 

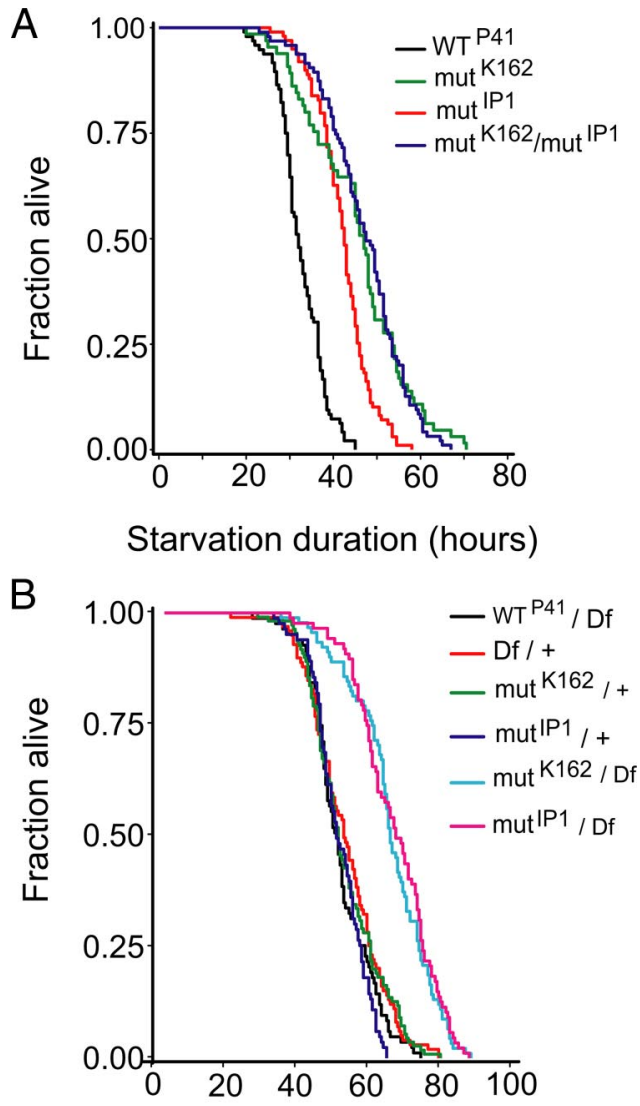

\section{Starvation duration (hours)}

Figure 8. Enhanced starvation resistance in slob mutant flies. Flies $<24 \mathrm{~h}$ old were aged for $3 \mathrm{~d}$. Individual flies were then transferred to tubes containing $1.5 \%$ agar only and monitored for survival at $0.5 \mathrm{~h}$ time intervals. Flies with activity counts $\geq 1$ in a 30 min bin were considered alive. $A$, Survival curves representing lifespan of different fly strains as a function of starvation duration. The fraction of animals alive is plotted against time under starvation condition. Compared with $\mathrm{WT}^{\mathrm{P} 41}$ flies, slob mutant (mut ${ }^{\mathrm{K} 162}$, mut $^{\mathrm{PP} 1}$, and transheterozygous mut ${ }^{\mathrm{K} 162}$ / mut $^{\mathrm{PP}}$ ) flies live significantly longer under starvation conditions. $\boldsymbol{B}$, Enhanced resistance to starvation maps close to the slob locus. To test whether the enhanced starvation resistance phenotype maps to the slob locus, we crossed WT ${ }^{\mathrm{P} 41}$, mut ${ }^{\mathrm{K} 162}$, and mut ${ }^{\mathrm{IP} 1}$ flies to a deficiency (Df) line spanning the slob locus. For heterozygous controls, $\mathrm{WT}^{\mathrm{P} 41}$, mut $^{\mathrm{K} 162}$, and mut ${ }^{\mathrm{IP} 1}$ were crossed to a $y w$ line to keep the same genetic background as their homozygous counterparts. Compared with $\mathrm{WT}^{\mathrm{P} 41} / \mathrm{Df}$ and heterozygous $\mathrm{Df} /+$, mut ${ }^{\mathrm{K} 162} /+$, and mut ${ }^{\mathrm{P} 1} /+$ control flies, both mut ${ }^{\mathrm{K} 162} / \mathrm{Df}$ and mut ${ }^{\mathrm{P} 1} / \mathrm{Df}$ flies exhibit greater resistance to starvation-induced death.

the function of the channel in the brains of living flies. The combination of in vivo macroscopic and single-channel recording with genetic manipulation provides a unique window into the participation of an ion channel regulatory protein complex in the control of neuronal physiology. Previous studies of the mammalian BK channel $\beta 4$ subunit, using in vitro recordings in brain slices from $\beta 4$ knock-out mice, have also demonstrated an essential role for a BK channel auxiliary subunit in neuronal physiology (Brenner et al., 2005).

It is evident from the data presented here that the level of expression of Slob is an important determinant of the excitability of the neurosecretory PI neurons in the fly brain. Slob expression is very different in different parts of the brain (Jaramillo et al., 2004, 2006), and is under circadian control (Jaramillo et al., 2004), so there is a clear physiological counterpart to the genetic manipulation of Slob expression that we exploit here. Because of these factors it is likely that Slob modulation of dSlo channel voltage dependence will vary from neuron to neuron and with
Table 1. slob mutants survive starvation longer than control flies

\begin{tabular}{|c|c|c|c|c|}
\hline Fly strain & $N$ & $\begin{array}{l}\text { Mean lifespan } \pm \\
\text { SEM (h) }\end{array}$ & $\begin{array}{l}\text { Half-life } \\
\text { (h) }\end{array}$ & $\begin{array}{l}\text { Increase in } \\
\text { half-life (\%) }\end{array}$ \\
\hline$W T^{P 41}$ & 96 & $32.5 \pm 0.5$ & 32.0 & \\
\hline mut $^{\mathrm{K} 162}$ & 65 & $45.4 \pm 1.4$ & $47.0^{* * *}$ & +47 \\
\hline mut $^{\mid P 1}$ & 99 & $42.1 \pm 0.6$ & $42.5^{* * *}$ & +33 \\
\hline mut $^{\mathrm{K} 162} /$ mut $^{\mathrm{IP} 1}$ & 95 & $47.1 \pm 0.9$ & $47.5^{* * *}$ & +48 \\
\hline \multicolumn{5}{|c|}{ Deficiency mapping } \\
\hline $\mathrm{WT}^{\mathrm{P} 41} / \mathrm{Df}$ & 83 & $49.1 \pm 1.0$ & 48.5 & \\
\hline $\mathrm{Df} /+$ & 99 & $50.6 \pm 1.0$ & 50.0 & \\
\hline mut $^{\mathrm{K} 162} /+$ & 110 & $50.3 \pm 1.0$ & 48.5 & \\
\hline mut ${ }^{\mathrm{IP} 1} /+$ & 83 & $48.9 \pm 0.8$ & 48.0 & \\
\hline mut $^{\mathrm{K} 162} / \mathrm{Df}$ & 91 & $63.2 \pm 1.2$ & $63.0^{* * *}$ & +30 \\
\hline mut ${ }^{I P 1} / D f$ & 87 & $64.3 \pm 1.2$ & $64.5^{* * *}$ & +32 \\
\hline
\end{tabular}

Control and experimental animals were assayed in parallel. The mean lifespan and half-lives of the different genotypes in the third and fourth columns, respectively, were determined using Statview (SAS) software. The half-life is the median, the time at which the fraction of live animals in each group is 0.5. The log-rank test (Lawless, 1982) was used to determine the statistical significance between different groups. ${ }^{* *}$ Statistically significant difference from control $\left(p<0.0001\right.$ ). Each of the mutant groups (mut ${ }^{\mathrm{K} 162}$, mut ${ }^{\mathrm{P} 1}$, and mut ${ }^{\mathrm{K} 162} / \mathrm{mut}^{\mathrm{P} 1}$ ) exhibits a significant increase in mean lifespan and half-life compared with the wild-type control $\left(\mathrm{WT}^{\mathrm{P} 41}\right)$. For deficiency-mapping data, four control groups (WT ${ }^{\mathrm{P} 41} / \mathrm{Df}$, Df/+, mut ${ }^{\mathrm{K} 162} /+$, and mut ${ }^{\mathrm{PP} 1} /+$ ) were used. As in the case of slob mutants, the two experimental groups, mut ${ }^{\mathrm{P} 1} / \mathrm{Df}$ and mut ${ }^{\mathrm{K} 162} / \mathrm{Df}$, are significantly different from each of the control groups. The percentage difference in half-life between the experimental and control groups in each dataset is shown as percentage increase in half-life in the fifth column.

time of day. This in turn will contribute to physiological variation in action potential duration and, ultimately, release of neurotransmitters and neurohormones such as DILPs from presynaptic terminals (Gho and Ganetzky, 1992; Warbington et al., 1996; Lee et al., 2008).

It is notable that two independent methods of knocking down Slob, P-element mutagenesis and targeted expression of transgenic Slob-RNAi, produce very similar effects on the whole-cell outward current. While neither of these genetic approaches by itself is immune from the possibility of experimental artifact, together the results suggest strongly that it is the decrease in Slob expression that accounts for the leftward shift in the $G-V$ relationship of the macroscopic current. Furthermore, transgenic expression of Slob57 in PI neurons (in a wild-type genetic background) shifts the $G-V$ relationship to the right and decreases the single-channel open probability, consistent with the genetic knockdown experiments and with the dSlo modulation observed upon coexpression of recombinant Slob57 and dSlo in transfected cells (Zeng et al., 2006). Finally, and perhaps most importantly, the changes in macroscopic current associated with both P-element mutagenesis and Slob-RNAi can be rescued by the targeted expression of Slob57. These several lines of evidence confirm that the electrophysiological phenotypes we describe here do indeed result from changes in the expression level of Slob. We cannot detect any differences between wild-type and Slobnull flies in the amount of dSlo protein in the fly heads (measured by Western blot; data not shown), indicating that the manipulation of Slob levels in vivo does not influence the expression of dSlo.

The total macroscopic outward current in the PI neurons is of course composed of currents flowing through a variety of potassium channels. To identify the particular neuronal potassium channel(s) that are influenced by Slob expression levels, we made use of the potassium channel blocker TEA. It has been shown that the presence of a particular tyrosine residue in the vicinity of the selectivity filter endows dSlo with a uniquely high sensitivity to TEA (Shen et al., 1994). In contrast, Shaker, Shab, Shaw, Shal, and dKCNQ channels in the fly lack this tyrosine residue and are much less sensitive to extracellular TEA (Kavanaugh et al., 1991). We found that $1 \mathrm{~mm}$ TEA reduces the total outward current in PI 
neurons by approximately one-third in wild-type flies, implying that this proportion of the neuronal outward current can be attributed to dSlo. The proportion of TEA-sensitive current increases to $\sim 45 \%$ in Slob-null as well as in Slob-RNAi transgenic flies, whereas it is only $\sim 15 \%$ of the outward current in flies in which transgenic Slob57 is expressed. This result is consistent with the idea that Slob selectively modulates the TEA-sensitive component of the total outward current, namely dSlo. This conclusion is confirmed convincingly by our unprecedented in vivo single-channel recordings, that demonstrate the modulation of the gating (but not of the single-channel conductance) of a largeconductance $(\sim 180 \mathrm{pS})$ potassium channel in Slob knockdown and transgenic Slob57-expressing flies. The single-channel conductance of dSlo is substantially greater than that of any other Drosophila potassium channel, so these data provide an unequivocal demonstration that neuronal dSlo channel gating is influenced profoundly by Slob. We have tested a number of other Drosophila channels in transfected cells and seen no evidence for their modulation by Slob (data not shown), and as we show here the $G-V$ relationship of the TEA-insensitive component of the outward current is not modulated by Slob. In addition, the quantitative changes in dSlo single-channel gating we describe here are sufficient to account for the modulation of the macroscopic outward current. These findings, together, argue strongly that Slob does not modulate neuronal potassium channels other than dSlo in vivo.

We have shown previously by heterologous expression that different Slob isoforms modulate dSlo in different ways (Zeng et al., 2005). The effects of Slob deletion and Slob knockdown that we present here are consistent with the modulatory actions of recombinant Slob57 (Zeng et al., 2006), suggesting that this is the predominant functional Slob isoform in the PI neurons. This finding is also consistent with the distribution of Slob isoforms determined by immunocytochemistry (Jaramillo et al., 2006).

Because Slo channels play a prominent role in action potential repolarization, we predicted that Slob knock-out would decrease action potential duration in the PI neurons, and this is indeed the case. Both dSlo and Slob are expressed highly in presynaptic terminals at the fly larval neuromuscular junction (Zhou et al., 1999), and it seems possible that Slob may influence neurotransmitter and neurohormone release by modulating the duration of action potentials at nerve terminals. What might be the physiological sequelae of such modulation? The PI neurons project to the CC and the dorsal blood vessel, allowing for the direct release of DILP into the circulating hemolymph (Rulifson et al., 2002), and the PI-CC system in insects is thought to be functionally homologous to the hypothalamic-pituitary axis in mammals (Veelaert et al., 1998). The CC is a neurohemal gland which produces adipokinetic hormone, $\mathrm{AKH}$, which is similar to mammalian glucagon, and together AKH and DILP regulate the levels of circulating sugars (Rulifson et al., 2002; Kim and Rulifson, 2004; Isabel et al., 2005). Thus it is particularly intriguing that flies lacking Slob survive significantly longer than wild-type flies during starvation, comparable to the increased resistance to starvation stress in flies with decreased insulin pathway signaling (Clancy et al., 2001; Broughton et al., 2005). It therefore will be of interest to determine whether dSlo and Slob influence the insulin signaling pathway, and whether changes in DILP release from the PI neurons underlie the increased survival during starvation observed in Slob mutant flies.

\section{References}

Adelman JP, Shen K-Z, Kavanaugh MP, Warren RA, Wu Y-N, Lagrutta A, Bond CT, North RA (1992) Calcium-activated potassium channels expressed from cloned complementary DNAs. Neuron 9:209-216.

Atkinson NS, Robertson GA, Ganetzky B (1991) A component of calciumactivated potassium channels encoded by the Drosophila slo locus. Science 253:551-555.

Brenner R, Chen QH, Vilaythong A, Toney GM, Noebels JL, Aldrich RW (2005) BK channel beta4 subunit reduces dentate gyrus excitability and protects against temporal lobe seizures. Nat Neurosci 8:1752-1759.

Broughton SJ, Piper MD, Ikeya T, Bass TM, Jacobson J, Driege Y, Martinez P, Hafen E, Withers DJ, Leevers SJ, Partridge L (2005) Longer lifespan, altered metabolism, and stress resistance in Drosophila from ablation of cells making insulin-like ligands. Proc Natl Acad Sci U S A 102:31053110.

Ceriani MF, Hogenesch JB, Yanovsky M, Panda S, Straume M, Kay SA (2002) Genome-wide expression analysis in Drosophila reveals genes controlling circadian behavior. J Neurosci 22:9305-9319.

Clancy DJ, Gems D, Harshman LG, Oldham S, Stocker H, Hafen E, Leevers SJ, Partridge L (2001) Extension of life-span by loss of CHICO, a Drosophila insulin receptor substrate protein. Science 292:104-106.

Claridge-Chang A, Wijnen H, Naef F, Boothroyd C, Rajewsky N, Young MW (2001) Circadian regulation of gene expression systems in the Drosophila head. Neuron 32:657-671.

Elkins T, Ganetzky B, Wu C-F (1986) A Drosophila mutation that eliminates a calcium-dependent potassium current. Proc Natl Acad Sci U S A 83:8415-8419.

Gho M, Ganetzky B (1992) Analysis of repolarization of presynaptic motor terminals in Drosophila larvae using potassium channel-blocking drugs and mutations. J Exp Biol 170:93-111.

Giordano E, Rendina R, Peluso I, Furia M (2002) RNAi triggered by symmetrically transcribed transgenes in Drosophila melanogaster. Genetics 160:637-648.

Isabel G, Martin JR, Chidami S, Veenstra JA, Rosay P (2005) AKHproducing neuroendocrine cell ablation decreases trehalose and induces behavioral changes in Drosophila. Am J Physiol Regul Integr Comp Physiol 288:R531-R538.

Jaramillo AM, Zheng X, Zhou Y, Amado DA, Sheldon A, Sehgal A, Levitan IB (2004) Pattern of distribution and cycling of SLOB, Slowpoke channel binding protein, in Drosophila. BMC Neurosci 5:3.

Jaramillo AM, Zeng H, Fei H, Zhou Y, Levitan IB (2006) Expression and function of variants of slob, slowpoke channel binding protein, in Drosophila. J Neurophysiol 95:1957-1965.

Kaneko M, Hall JC (2000) Neuroanatomy of cells expressing clock genes in Drosophila: transgenic manipulation of the period and timeless genes to mark the perikarya of circadian pacemaker neurons and their projections. J Comp Neurol 422:66-94.

Kavanaugh MP, Varnum MD, Osborne PB, Christie MJ, Busch AE, Adelman JP, North RA (1991) Interaction between tetraethylammonium and amino acid residues in the pore of cloned voltage-dependent potassium channels. J Biol Chem 266:7583-7587.

Kim SK, Rulifson EJ (2004) Conserved mechanisms of glucose sensing and regulation by Drosophila corpora cardiaca cells. Nature 431:316-320.

Kramer JM, Davidge JT, Lockyer JM, Staveley BE (2003) Expression of Drosophila FOXO regulates growth and can phenocopy starvation. BMC Dev Biol 3:5.

Krauthamer V (1985) Morphology of identified neurosecretory and nonneurosecretory cells in the cockroach pars intercerebralis. J Exp Zool 234:221-230.

Lagrutta A, Shen K-Z, North RA, Adelman JP (1994) Functional differences among alternatively spliced variants of Slowpoke, a Drosophila calciumactivated potassium channel. J Biol Chem 269:20347-20351.

Lawless J (1982) Models and methods for lifetime data. New York: Wiley.

Lee G, Park JH (2004) Hemolymph sugar homeostasis and starvationinduced hyperactivity affected by genetic manipulations of the adipokinetic hormone-encoding gene in Drosophila melanogaster. Genetics 167:311-323.

Lee J, Ueda A, Wu CF (2008) Pre- and post-synaptic mechanisms of synaptic strength homeostasis revealed by slowpoke and shaker $\mathrm{K}(+)$ channel mutations in Drosophila. Neuroscience 154:1283-1296.

Levitan IB (2006) Signaling protein complexes associated with neuronal ion channels. Nat Neurosci 9:305-310. 
Lin Y, Han M, Shimada B, Wang L, Gibler TM, Amarakone A, Awad TA, Stormo GD, Van Gelder RN, Taghert PH (2002) Influence of the period-dependent circadian clock on diurnal, circadian, and aperiodic gene expression in Drosophila melanogaster. Proc Natl Acad Sci U S A 99:9562-9567.

McDonald MJ, Rosbash M (2001) Microarray analysis and organization of circadian gene expression in Drosophila. Cell 107:567-578.

Raffaelli G, Saviane C, Mohajerani MH, Pedarzani P, Cherubini E (2004) BK potassium channels control transmitter release at CA3-CA3 synapses in the rat hippocampus. J Physiol 557:147-157.

Rubin GM, Spradling AC (1982) Genetic transformation of Drosophila with transposable element vectors. Science 218:348-353.

Rulifson EJ, Kim SK, Nusse R (2002) Ablation of insulin-producing neurons in flies: growth and diabetic phenotypes. Science 296:1118-1120.

Sarov-Blat L, So WV, Liu L, Rosbash M (2000) The Drosophila takeout gene is a novel molecular link between circadian rhythms and feeding behavior. Cell 101:647-656.

Schopperle WM, Holmqvist MH, Zhou Y, Wang J, Wang Z, Griffith LC, Keselman I, Kusinitz F, Dagan D, Levitan IB (1998) Slob, a novel protein that interacts with the slowpoke calcium-dependent potassium channel. Neuron 20:565-573.

Shao LR, Halvorsrud R, Borg-Graham L, Storm JF (1999) The role of BKtype $\mathrm{Ca} 2+$-dependent $\mathrm{K}+$ channels in spike broadening during repetitive firing in rat hippocampal pyramidal cells. J Physiol 521:135-146.

Shen K-Z, Lagrutta A, Davies NW, Standen NB, Adelman JP, North RA (1994) Tetraethylammonium block of Slowpoke calcium-activated potassium channels expressed in Xenopus oocytes: evidence for tetrameric channel formation. Pflugers Arch 426:440-445.
Siegmund T, Korge G (2001) Innervation of the ring gland of Drosophila melanogaster. J Comp Neurol 431:481-491.

Sokolove PG, Loher W (1975) Role of eyes, optic lobes, and pars intercerebralis in locomotory and stridulatory circadian rhythms of Teleogryllus commodus. J Insect Physiol 21:785-799.

Ueda HR, Matsumoto A, Kawamura M, Iino M, Tanimura T, Hashimoto S (2002) Genome-wide transcriptional orchestration of circadian rhythms in Drosophila. J Biol Chem 277:14048-14052.

Veelaert D, Schoofs L, De Loof A (1998) Peptidergic control of the corpus cardiacum-corpora allata complex of locusts. Int Rev Cytol 182:249-302.

Vergara C, Latorre R, Marrion NV, Adelman JP (1998) Calcium-activated potassium channels. Curr Opin Neurobiol 8:321-329.

Warbington L, Hillman T, Adams C, Stern M (1996) Reduced transmitter release conferred by mutations in the slowpoke-encoded $\mathrm{Ca} 2(+)$ activated $\mathrm{K}+$ channel gene of Drosophila. Invert Neurosci 2:51-60.

Zaretsky M, Loher W (1983) Anatomy and electrophysiology of individual neurosecretory cells of an insect brain. J Comp Neurol 216:253-263.

Zeng H, Weiger TM, Fei H, Jaramillo AM, Levitan IB (2005) The amino terminus of Slob, Slowpoke channel binding protein, critically influences its modulation of the channel. J Gen Physiol 125:631-640.

Zeng H, Weiger TM, Fei H, Levitan IB (2006) Mechanisms of two modulatory actions of the channel-binding protein Slob on the Drosophila Slowpoke calcium-dependent potassium channel. J Gen Physiol 128:583-591.

Zhou Y, Schopperle WM, Murrey H, Jaramillo A, Dagan D, Griffith LC, Levitan IB (1999) A dynamically regulated 14-3-3, Slob, and Slowpoke potassium channel complex in Drosophila presynaptic nerve terminals. Neuron 22:809-818. 